\title{
WORKLOAD BOUNDS IN FLUID MODELS WITH PRIORITIES
}

\author{
Arthur W. Berger ${ }^{1}$ and Ward Whitt ${ }^{2}$
}

December 17, 1998

\footnotetext{
${ }^{1}$ Lucent Technologies, Holmdel, NJ 07733-3030; awberger@lucent.com

${ }^{2}$ AT\&T Labs, Room A117, Shannon Laboratory, 180 Park Avenue, Florham Park, NJ 07932-0971; wow@research.att.com
} 


\begin{abstract}
In this paper we establish upper and lower bounds on the steady-state per-class workload distributions in a single-server queue with multiple priority classes. Motivated by communication network applications, the model has constant processing rate and general input processes with stationary increments. The bounds involve corresponding quantities in related models with the firstcome first-served discipline. We apply the bounds to support a new notion of effective bandwidths for multi-class systems with priorities. We also apply the lower bound to obtain sufficient conditions for the workload distributions to have heavy tails.
\end{abstract}

Key words: priority queues, stochastic fluid models, bounds, admission control, effective bandwidths, large-buffer asymptotics, ATM, heavy-tailed distributions, long-tailed distributions 


\section{Introduction}

Motivated by the desire to model asynchronous transfer mode (ATM) switches and internet protocol (IP) routers supporting multiple priority classes, we consider a stochastic fluid queue with unlimited buffer space, constant release rate and $m$ priority classes. We allow the input for each class to arrive in an arbitrary manner. Our main assumptions are that the $m$ single-class cumulative input processes are mutually independent and that each has stationary increments. We want to allow general stationary input processes in order to be able to represent traffic complexity as observed in many recent traffic measurements; e.g., see Cáceres, Danzig, Jamin and Mitzel [6], Leland, Taqqu, Willinger and Wilson [17] and Feldmann, Gilbert, Willinger and Kurtz [12].

We assume that the priority service discipline is preemptive-resume, which in our fluid context means that the constant output rate available at any instant is applied to the highest-priority work waiting or arriving at that instant. Note that in the application to packet networks the transmission of a packet is not pre-empted; however, the resulting inaccuracy of assuming pre-emptive resume is well with the inaccuracy of the overall traffic model, particularly in the region of interest of many packets in queue. Within each priority class, work is served in a first-come first-served (FCFS) order. With this priority discipline, it actually suffices to consider only two priority classes. From the perspective of any class, all lower-priority classes can be ignored, while all higher-priority classes can be lumped together. Thus, without loss of generality, we consider only two priority classes, with class 1 having priority over class 2 . Since class 1 experiences a FCFS system, we are primarily interested in the steady-state workload (buffer content) and waiting time for the low-priority class 2. The waiting time is a virtual waiting time; in particular, the waiting time at time $t$ is the time until a potential additional infinitesimal particle of fluid arriving at time $t$ would be served (processed). The low-priority waiting-time at any instant is at least as large as the total workload at that instant divided by the processing rate; it often is strictly larger because subsequent high-priority input has priority over waiting low-priority work.

Our main results for the low-priority workload and waiting time are upper and lower bounds in terms of associated stochastic fluid models with the FCFS service discipline. These bounds allow us to apply results for FCFS systems to bound and approximate the low-priority steady-state workload and waiting time.

Our bounds have many possible applications. We developed them in order to extend the concept of effective bandwidths for admission control to settings with multiple priority classes. That 
application of the bounds is described in [2], so we will be brief here. The notion of effective bandwidths was originally developed for the FCFS discipline. The general idea is to assign an effective bandwidth $e_{i}$ to each connection of type $i$. Then a vector $\left(n_{1}, \ldots, n_{I}\right)$, where $n_{i}$ is the number of connections of type $i$, is deemed feasible for a system with capacity (i.e., available bandwidth or constant processing rate) $c$ if

$$
\sum_{i \in I} n_{i} e_{i} \leq c
$$

The associated admissible set with a single linear boundary greatly simplifies engineering; e.g., it makes it possible to apply stochastic-loss-network (generalized-Erlang) models, as in Ross [21], for capacity planning. A theoretical basis for the notion of effective bandwidths and the linearadmissible-set structure for the FCFS discipline has been provided by large-buffer asymptotics (large deviations theory); e.g., see Chang and Thomas [7], de Veciana, Kesidis and Walrand [9], Kelly [15] and Whitt [27] for reviews.

As discussed in [2], a corresponding large-buffer asymptotics can be developed for stochastic fluid queues with priorities. The resulting admissible set has a constraint for each priority class. That by itself presents no major problem, but unfortunately these constraints are in general nonlinear. Losing the linearity causes the notion of effective bandwidths to lose much of its appeal. Fortunately, the FCFS bounds introduced here can help. The FCFS bounds produce approximating admissible sets that do have linear boundaries. In particular, the admissible set associated with the upper (lower) bound has linear boundaries and is contained in (contains) the exact admissible set with nonlinear boundaries; i.e., the upper bound on the workload tail probabilities is conservative, leading to a smaller admissible set. In [2] we suggest the conservative upper bound for the steady-state workload and the associated smaller admissible set as the preferred approximation.

Even more important than the candidate approximations for effective bandwidths, we believe, is the proposed structure for the admissible set with multiple priority classes. Regardless of the method used to define effective bandwidths, the analysis suggests that there should be a linear constraint associated with each priority class. This linear-admissible-set structure implies a new notion of effective bandwidths, where a given connection is associated with multiple effective bandwidths: one for the priority level of the given connection and one for each lower priority level. We have made a case for this general admissible-set structure, without referring to large-buffer asymptotics in [3]. This approximating admissible set was also suggested by Kulkarni and Gautam [16], but they obtained it by examining the exact admissible set, rather than from general bounds on the steady-state workloads. 
Here is how the rest of this paper is organized: In Section 2 we define the stochastic processes of interest in the two-priority model. In Section 3 we show how to construct stationary versions of the stochastic processes defined in Section 2. In Section 4 we apply the stationary versions together with previous large-deviations results in [13], [27] to establish the exact large-buffer deviation result.

In Section 5 we establish an exact relation between the low-priority waiting time and the total workload under the assumption that the high-priority input has stationary and independent increments, which is applied in our bounds and is also directly useful. In Section 6 we establish the lower bound on the low-priority steady-state workload, which we call the reduced-service-rate bound. In Section 7 we combine this bound with another lower bound in [8] to obtain general sufficient conditions for the low-priority workload to have a heavy-tailed distribution. In Section 8 we establish the upper bound on the steady-state workload, which we call the empty-buffer bound. Finally, in Section 9 we consider an illustrative numerical example.

We close this introduction by mentioning other related work. Exact analyses of priority models with extra Markovian assumptions have been done by Sugahara, Takine, Takahashi and Hasegawa [23], Takine and Hasegawa [24] and Zhang [29]. Zhang [29] finds an exact solution for a Markov modulated fluid model with priorities, and Elwalid and Mitra [11] develop an approximation. With admission control, these approaches offer the possibility of calculating the feasible set more accurately, but at the expense of losing the more elementary effective bandwidth approach with linear constraint boundaries.

\section{The General Fluid Model with Priorities}

In this section we define the basic stochastic processes in the general fluid model with priorities. By "fluid model" we mean that work is processed continuously at a constant rate as if it were a fluid. We let work arrive in an arbitrary manner; i.e., it could arrive continuously or in jumps. There is a single server (or buffer) with unlimited waiting space. The specific priority discipline is preemptive resume; i.e., higher priority work immediately preempts lower priority work, and lower priority work resumes service where it stopped when it regains access to the server.

In general, there may be $m$ priority classes, but it suffices to consider only two. Hence, let there be two priority classes, with class 1 having preemptive priority over class 2 . Let class $i$ have required work arrive according to the stochastic process $\left\{A_{i}(t): t \geq 0\right\}$; i.e., $A_{i}(t)$ is the input for class $i$ over the interval $[0, t]$. (The process $A_{i}(t)$ might be the superposition of arrival processes from independent sources.) We assume that $A_{i}(t)$ has nondecreasing sample paths. Let the work 
be processed continuously at rate $c$ in order of the priority. Thus, assuming that the system starts with initial workload $V_{i}(0)$ for class $i$ at time 0 , the workload for class $i$ at time $t$ can be defined by

$$
V_{i}(t)=V_{i}(0)+X_{i}(t)-\inf _{0 \leq s \leq t}\left\{\min \left\{0, V_{i}(0)+X_{i}(s)\right\}\right\}, \quad t \geq 0
$$

where

$$
\begin{aligned}
X_{i}(t) & =A_{i}(t)-S_{i}(t), \\
S_{1}(t) & =c t, \\
S_{2}(t) & =c t-D_{1}(t), \\
D_{i}(t) & =A_{i}(t)+V_{i}(0)-V_{i}(t),
\end{aligned}
$$

with $A_{i}(0)=0$ for all $i$. The processes $\left\{S_{i}(t): t \geq 0\right\}$ in (2.3) and (2.4) are the server-availability processes; i.e., $S_{i}(t)$ is the total potential processing that can be done for class $i$ in the interval $[0, t]$. The maximum server processing rate is the capacity or available bandwidth $c$. Clearly, (2.3) holds for the high priority class. The processes $\left\{D_{i}(t): t \geq 0\right\}$ are the departure (output) processes; i.e., the output in completed work during the interval $[0, t]$. The output $D_{i}(t)$ is the input over $[0, t]$, plus the initial work, minus what is present at time $t$, as indicated in (2.5). For $i=2$, the server-availability process can be defined in terms of the departure process of the high priority class by (2.4). Finally, the process $\left\{X_{i}(t): t \geq 0\right\}$ in (2.2) is the (cumulative) net input process for class $i$, in terms of which the workload process is defined by the usual one-dimensional reflection map in (2.1).

It is important to distinguish between the workload process and the waiting-time process. The workload $V_{i}(t)$ is the class- $i$ work in the system at time $t$ (e.g., in units of bits), while the (virtual) waiting time $W_{i}(t)$ is the time required to clear the workload $V_{i}(t)$ at time $t$ (not counting any class- $i$ input after time $t$ ). However, the class- 2 waiting time at time $t$ depends on the class- 1 input after time $t$. We can define the class- $i$ waiting time $W_{i}(t)$ by

$$
W_{i}(t)=\inf \left\{u: u \geq 0 \text { and } S_{i}(t+u)-S_{i}(t) \geq V_{i}(t)\right\}, \quad t \geq 0
$$

Combining (2.3) and (2.6), we see that $W_{1}(t)=V_{1}(t) / c$, as it should, but in general we only have

$$
W_{2}(t) \geq V_{2}(t) / c \text { for all } \mathrm{t}
$$

Indeed, the low-priority waiting time $W_{2}(t)$ can be much greater than the scaled low-priority workload $V_{2}(t) / c$ if the server is frequently occupied with high-priority work. 
If customers arrive at random times and bring service requirements, then the processes $A_{i}$ are pure jump processes, having jumps up equal to the service times and $W_{i}(t)$ is the virtual waiting time process (the time a potential arrival at time $t$ would have to wait before beginning service). If there are arrivals at time $t$, then $W_{i}(t)$ is the time required for all these arrivals to complete service. With Poisson arrivals, the steady-state virtual waiting time coincides with the steady-state actual waiting time (before beginning service) seen by arrivals, by the Poisson Arrivals See Time Averages (PASTA) property; see Wolff [28].

If work arrives continuously at a random rate, work can be processed without there being any work in the buffer. This will occur whenever the buffer is empty and the input rate is positive but less than the output rate $c$.

\section{Constructing Stationary Versions}

In Section 2 we indicated how to define the stochastic processes of interest, with general initial conditions. Now we construct stationary verions of these processes, which describe the system in equilibrium or steady state. For background, see Baccelli and Brémaud [1] and Borovkov [4].

Indeed, so far we have made no stochastic assumptions. Now we assume that the stochastic processes $A_{i}$ are mutually independent processes, each of which has stationary and ergodic increments with

$$
\lim _{t \rightarrow \infty} \frac{A_{i}(t)}{t}=c \rho_{i} \quad \text { w.p.1 for each } \quad i
$$

where $\rho \equiv \rho_{1}+\rho_{2}<1$. The stability condition $\rho<1$ ensures that the average rate that work enters is less than the processing rate $c$. This condition enables us to construct stationary versions of all the processes, as we show below. To treat the high-priority workload, we can use standard arguments as in Section 6 of Borovkov [4] and Chapter 2 of Baccelli and Brémaud [1]. However, it is more complicated to obtain a stationary version of the low-priority workload, because the low-priority workload depends on the high-priority departure process, as can be seen from (2.4). Thus, we successively construct stationary versions of the stochastic processes $V_{1}, D_{1}, V_{2}, D_{2}$ and $W_{2}$. (Since $W_{1}(t)=V_{1}(t) / c$, nothing special need be done for $W_{1}$.)

For this purpose, let $\Rightarrow$ denote convergence in distribution. First, as is customary, we extend the processes $A_{i}$ to be over $(-\infty, \infty)$ with stationary increments, but still keep the convention that $A_{i}(0)=0$ for $i=1,2$, which implies that $X_{i}(0)=0$. Then the stationary increments condition on 
$A_{1}$ implies that

$$
\hat{V}_{1}(t) \equiv \sup _{0 \leq s \leq t}\left\{X_{1}(t)-X_{1}(s)\right\} \stackrel{d}{=} \sup _{0 \leq s \leq t} X_{1}(-s), \quad t \geq 0
$$

i.e., the stationarity in $X_{1}$ allows us to construct the steady-state workload as the simple maximum of the reverse-time net input process, with initial workload 0. Since the final supremum in (3.2) is nondecreasing in $t, V_{1}(t) \Rightarrow \hat{V}_{1}$ as $t \rightarrow \infty$. Condition (3.1) for $i=1$, implies that $X_{1}(t) / t \rightarrow c\left(\rho_{1}-1\right)$ and $X_{1}(t) \rightarrow-\infty$ as $t \rightarrow \infty$ w.p.1. Hence, the steady-state high-priority workload is

$$
V_{1} \stackrel{d}{=} \sup _{s \geq 0} X_{1}(-s)<\infty \quad \text { w.p.1. }
$$

More generally, there is a stationary version of the stochastic process $\left\{V_{1}(t): t \geq 0\right\}$, denoted by $\left\{V_{1}^{*}(t): t \geq 0\right\}$, with

$$
V_{1}^{*}(t)=\sup _{s \leq t}\left\{X_{1}(t)-X_{1}(s)\right\}, \quad t \geq 0 ;
$$

i.e., the random vector $\left(V_{1}^{*}\left(t_{1}+h\right), \ldots, V_{1}^{*}\left(t_{k}+h\right)\right)$ has a distribution independent of $h$ for all $k$ and all $k$-tuples $\left(t_{1}, \ldots, t_{k}\right)$; see Chapter 1 of Borovkov [4].

Given the stationary process $\left\{V_{1}^{*}(t): t \geq 0\right\}$, the associated stationary departure process $\left\{D^{*}(t): t \geq 0\right\}$ defined by $(2.5)$ is

$$
D_{1}^{*}(t)=A_{1}(t)+V_{1}^{*}(0)-V_{1}^{*}(t)
$$

i.e., it has stationary increments. (However, note that in general variables $A_{1}(t)$ and $V_{1}^{*}(0)$ are not independent.) This in turn makes the associated stochastic process $S_{2}^{*}(t)$ and $X_{2}^{*}(t)$ have stationary increments. Given that $V_{1}^{*}(t)$ has a proper distribution for each $t,(3.1)$ implies that $t^{-1} V_{1}^{*}(t) \rightarrow 0$ w.p.1 as $t \rightarrow \infty$. Since this is an important technical point, we state it as a proposition and prove it.

Proposition 3.1. Under the assumptions above,

$$
\frac{V_{1}^{*}(t)}{t} \rightarrow 0 \quad \text { w.p.1 as } t \rightarrow \infty
$$

Proof. The limit $t^{-1} X(t) \rightarrow c\left(\rho_{1}-1\right)$ as $t \rightarrow \infty$ w.p.1 implies the stronger functional limit $n^{-1} X(n t) \rightarrow c\left(\rho_{1}-1\right) t$ w.p.1 as $n \rightarrow \infty$, with convergence being uniform in $t$ over bounded intervals; see Theorem 4 of Glynn and Whitt [13]. Then apply the continuous mapping theorem with the reflection map and general initial condition $V_{1}^{*}(0)$ as in Theorem 6.4 (iii) of Whitt [26] to get $n^{-1} V_{1}(n t) \rightarrow 0$ w.p.1 as $n \rightarrow \infty$, again uniform in $t$ over bounded intervals, which implies the desired result. 
Given Proposition 3.1, (3.1) and (3.5) imply that

$$
\frac{D_{1}^{*}(t)}{t} \rightarrow c \rho_{1} \quad \text { as } t \rightarrow \infty \text { w.p.1 }
$$

so that

$$
\frac{X_{2}^{*}(t)}{t} \rightarrow c(\rho-1) \quad \text { as } t \rightarrow \infty \text { w.p.1 }
$$

Hence, we can repeat the construction above to construct a stationary version $\left\{V_{2}^{*}(t): t \geq 0\right\}$ of the stochastic process $\left\{V_{2}(t): t \geq 0\right\}$ with $V_{2}^{*}(t)<\infty$ w.p.1.

Given that $V_{2}^{*}(t)$ is proper, we can apply Proposition 3.1 again to deduce that $t^{-1} V_{2}^{*}(t) \rightarrow 0$ w.p.1 as $t \rightarrow \infty$. Then conditions (2.5) and (3.1) imply that

$$
\frac{D_{2}^{*}(t)}{t} \rightarrow c \rho_{2} \quad \text { as } t \rightarrow \infty \quad \text { w.p.1 }
$$

Finally, we obtain a stationary version $W_{2}^{*}$ of $W_{2}(t)$ defined in terms of $\left(S_{2}^{*}, V_{2}^{*}\right)$ as in $(2.6)$. The supporting theorem is the continuous-time analog of Proposition 6.6 on p. 105 of Breiman [5]. Let $V_{i}$ and $W_{i}$ be random variables with the steady-state distributions of $V_{i}(t)$ and $W_{i}(t)$.

\section{The Large Deviations Result}

Suppose that we have criteria on the steady-state workload tail probabilities for each priority class that we want satisfied, e.g.,

$$
P\left(V_{i}>b_{i}\right) \leq p_{i} \quad \text { for each priority class } i
$$

We think of this tail probability constraint as a surrogate for the constraint that the probability of a buffer overflow from a buffer of size $b_{i}$ be less than $p_{i}$ for class $i$. It is natural to use the workload in (4.1) instead of the waiting time or sojourn time if we are interested in the probabilities of buffer overflows. Then the tail probability $P\left(V_{i}>b_{i}\right)$ is an approximation for the probability that class- $i$ work will overflow a buffer of size $b_{i}$ when there are separate buffers dedicated to each priority class.

With criteria such as (4.1), we can use the notion of effective bandwidths as in [27] to develop an admission control procedure for sources of each priority class. However, with priorities, we must proceed recursively over the priority classes. The possibilities for lower priorities depend on the high-priority sources in service.

Let there be multiple sources of each of the two priority classes. Let the sources be indexed by the pair $(i, j)$, representing source type $j$ of priority class $i$. Let $J_{i}$ be the number of source types for priority class $i$. Let $A_{i j}(t)$ be the arrival process of an $(i, j)$ source. Let $A_{i j}(t)$ be a general 
input process with nondecreasing sample paths; e.g., $A_{i j}(t)$ represents the number of bits to arrive at a network node from an $(i, j)$ source during the interval $[0, t]$. Let

$$
\psi_{A_{i j}}(\theta)=\lim _{t \rightarrow \infty} t^{-1} \log E e^{\theta A_{i j}(t)}
$$

be the single-source arrival-process asymptotic-decay-rate functions (cumulant generating functions) as in (1.10) of [27] (without assuming here that $A_{i j}$ has rate 1). We assume that these decay-rate functions are well defined. Given mutually independent sources, with $n_{i j}(i, j)$-sources, we can form associated aggregate asymptotic-decay-rate functions

$$
\psi_{A_{i}}(\theta)=\sum_{j=1}^{J_{i}} \psi_{A_{i j}}(\theta) n_{i j}
$$

for priority class $i$. We give explicit formulas for asymptotic-decay-rate functions in [27] and Section IV of $[2]$.

Similarly, we can form the associated asymptotic-decay-rate functions for the server-availability processes by letting

$$
\psi_{S_{i}}(\theta)=\lim _{t \rightarrow \infty} t^{-1} \log E e^{\theta S_{i}(t)} .
$$

For the high-priority class, $S_{1}(t)=c t, t \geq 0$, so that

$$
\psi_{S_{1}}(\theta)=c \theta
$$

However, the low-priority service-availability process $S_{2}(t)$ is more complicated, but by (2.4) we can express it in terms of $\psi_{D_{1}}(\theta)$,

We now show how to use the asymptotic-decay-rate functions to define a notion of effective bandwidths for $(i, j)$ sources using criterion (4.1). The analysis of effective bandwidths here is the natural extension of the effective bandwidths for the queue length process in [27], just as in Section 5 of [27]. On p. 75 of [27], the processes, $A_{i}(t)$ and $S_{i}(t)$ are counting processes, and the key equations are (1.12) and (1.17). (In [27] and [14] three essentially equivalent processes were studied for the standard queueing model: the queue length process, the workload process and the discrete-time waiting-time sequence, with each process being essentially a reflection of a net input process, and with each process having its own effective-bandwidth equation. Here, with the more general processes $A_{i}(t)$ and $S_{2}(t)$, we focus only on the generalization of the queue length process with net input process $X_{i}(t)=A_{i}(t)-S_{i}(t)$, as in Section 5 of [27].)

The notion of effective bandwidths is based on an exponential approximation for the workload tail probabilities,

$$
P\left(V_{i}>b_{i}\right) \approx e^{-\eta_{i} b_{i}},
$$


assuming that $b_{i}$ is relatively large. Given (4.1) and (4.6), we want to choose $\eta_{i}$ in (4.6) so that

$$
\eta_{i} \geq \eta_{i}^{*} \equiv \frac{-\log p_{i}}{b_{i}}
$$

The theoretical basis for the exponential approximation (4.6) is an asymptotic result for the workload tail probability $P\left(V_{i}>t\right)$ as $t \rightarrow \infty$, Theorem 10 of [27], which is a minor modification of Theorem 4 of Glynn and Whitt [14]. We restate it here in the context of our priority model.

Theorem 4.1. Consider the general stationary two-priority queueing model in Section 3. If there exits a function $\psi_{X_{i}}$ and positive constants $\theta_{i}^{*}$ and $\epsilon$ such that

$$
t^{-1} \log E e^{\theta\left[A_{i}(t)-S_{i}(t)\right]} \rightarrow \psi_{X_{i}}(\theta)=\psi_{A_{i}}(\theta)+\psi_{S_{i}}(-\theta) \text { as } t \rightarrow \infty \text { for }\left|\theta-\theta_{i}^{*}\right|<\epsilon
$$

with $\psi_{X_{i}}$ finite in a neighborhood of $\theta_{i}^{*}$ and differentiable at $\theta_{i}^{*}$ with

$$
\psi_{X_{i}}\left(\theta_{i}^{*}\right) \equiv \psi_{A_{i}}\left(\theta_{i}^{*}\right)+\psi_{S_{i}}\left(-\theta_{i}^{*}\right)=0
$$

and $\psi_{X_{i}}^{\prime}\left(\theta_{i}^{*}\right)>0$, then

$$
t^{-1} \log P\left(V_{i}>t\right) \rightarrow-\theta_{i}^{*} \text { as } t \rightarrow \infty
$$

In Theorem 10 of [27] there is a condition that there exists a constant $M$ such that $S_{i}(\delta) \leq M$ for all sufficiently small $\delta$. That condition is satisfied here because $S_{i}(t) \leq c t$ for the model in Section 2. As shown in Duffield and O'Connell [10], the conditions can be weakened somewhat. The differential of $\psi_{X_{i}}(\theta)$ can be omitted and, instead of a root to (4.9), it suffices to have

$$
\theta_{i}^{*}=\sup \left\{\theta>0: \psi_{X_{i}}(\theta) \leq 0\right\}
$$

but (4.9) is the usual case.

We now apply Theorem 4.1 to develop notions of effective bandwidths and effective capacities for the two priority classes. Let the effective bandwidth of an $(i, j)$ source be

$$
e_{i j}=\frac{\psi_{A_{i j}}\left(\eta_{i}^{*}\right)}{\eta_{i}^{*}}
$$

For class 1 , this is the customary procedure. Note that $e_{i j}$ depends only on the source- $j$ input process $\left\{A_{i j}(t): t \geq 0\right\}$ of priority $i$ (not on $A_{i k}(t)$ for $k \neq j$ ).

Let the effective capacity available for class $i$ be

$$
C_{i}=\frac{-\psi_{S_{i}}\left(-\eta_{i}^{*}\right)}{\eta_{i}^{*}}
$$


We then say that the collection of sources consisting of $n_{i j}$ sources of type $j, 1 \leq j \leq J_{i}$, are feasible, given the aggregate input process for higher priorities, if

$$
\sum_{j=1}^{J_{i}} e_{i j} n_{i j} \leq C_{i}
$$

Note that the admissible set in (4.14) is linear for each $i$, but the low-priority (class-2) admissible set depends upon the high-priority sources in service via the effective capacity $C_{2}$.

The admissibility criterion (4.14) holds if and only if

$$
\sum_{j=1}^{J_{i}} \psi_{A_{i j}}\left(\eta_{i}^{*}\right) n_{i j}+\psi_{S_{i}}\left(-\eta_{i}^{*}\right) \leq 0 .
$$

This is what we want, because then the prevailing class $i$ decay rate $\theta_{i}^{*}$ will then exceed $\eta_{i}^{*}$ defined in (4.7) by virtue of Theorem 4.1. To see this, note that $\psi_{A_{i}}$ and $\psi_{S_{i}}$ are increasing and convex, which implies that $-\psi_{S_{i}}(-\theta)$ is increasing and concave, so that

$$
\psi_{A_{i}}(\theta) \leq-\psi_{S_{i}}(-\theta)
$$

for $0 \leq \theta \leq \theta_{i}^{*}$ and

$$
\psi_{A_{i}}(\theta) \geq-\psi_{S_{i}}(-\theta)
$$

for $\theta \geq \theta_{i}^{*}$. Hence, $\theta_{i}^{*} \geq \eta_{i}^{*}$ as claimed. (This makes $p_{i} \approx e^{-\eta_{i}^{*} b_{i}}>e^{-\theta_{i}^{*} b_{i}}$.)

Note that the effective capacities for classes 1 and 2 simplify to nice, intuitive expressions. Since

$$
\begin{aligned}
\psi_{S_{i}}(\theta) & =c \theta \text { and } \psi_{S_{2}}(\theta)=c \theta+\psi_{D_{1}}(-\theta) \\
C_{1} & =\frac{-\psi_{S_{1}}\left(-\eta_{1}^{*}\right)}{\eta_{1}^{*}}=\frac{c \eta_{1}^{*}}{\eta_{1}^{*}}=c
\end{aligned}
$$

and

$$
C_{2}=\frac{c \eta_{2}^{*}-\psi_{D_{1}}\left(\eta_{2}^{*}\right)}{\eta_{2}^{*}}=c-\frac{\psi_{D_{1}}\left(\eta_{2}^{*}\right)}{\eta_{2}^{*}},
$$

where $\psi_{D_{1}}(\theta)$ is given below in (4.19). We call $\psi_{D_{1}}\left(\eta_{2}^{*}\right) / \eta_{2}^{*}$ in (4.18) the effective capacity for class 2 used up by class 1 .

To proceed further, from (4.18) we see that we need to determine the asymptotic-decay-rate function $\psi_{D_{1}}(\theta)$ for the high-priority departure process, but this is where the nonlinearity comes in. Under regularity conditions, see [2], [19] and references cited there,

$$
\psi_{D_{1}}(\theta)= \begin{cases}\psi_{A_{1}}(\theta), & \theta<\hat{\theta} \\ \psi_{A_{1}}(\hat{\theta})+c(\theta-\hat{\theta}), & \theta>\hat{\theta}\end{cases}
$$


with $\hat{\theta}$ determined by the equation

$$
\psi_{A_{1}}^{\prime}(\hat{\theta})=c
$$

Our two bounds will avoid the nonlinearity in (4.19). For further discussion, see [2].

\section{An Exact Result for a Special Case}

In this section, under an additional assumption, we obtain an exact relation between the lowpriority waiting time $W_{2}$ and the total workload $V$. Since $V$ is the same as for the FCFS discipline, this establishes an important connection to FCFS models. This relation can provide the basis for both exact results and approximations for $W_{2}$. The extra assumption is that the class- 1 input process $A_{1}$ has independent as well as stationary increments. Such an assumption might be appropriate for an ATM switch if the high-priority class is predominantly constant-bit-rate (CBR) traffic. Due to network jitter and lack of synchronization, it may be reasonable to model the CBR input as a Poisson process.

We exploit the fact that $W_{2}$ is the class- 1 first passage time to 0 starting from the steady-state workload of both classes. Let $T_{x 0}^{(1)}$ denote the class- 1 first passage time from $x$ to 0 . This first passage time accounts for future random input and the constant output rate $c$. The independentincrements property makes the future inputs, starting in $V$ independent of $V$, which we understand to hold when we write $T_{V 0}^{(1)}$.

Since we already have assumed that $A_{1}$ has stationary increments, the independent-increments assumption makes $A_{1}$ a subordinator or, equivalently, a Lévy process with nonnegative nondecreasing sample paths, as on p. 69 of Prabhu [18]. A subordination is characterized by its characteristic Laplace exponent $\phi(s)$, where

$$
E e^{-s A(t)}=e^{-t \phi(s)}, \quad t>0
$$

Theorem 5.1. With the general stationary model, if in addition the high-priority input process $A_{1}$ has independent increments, then

$$
\begin{gathered}
W_{2} \stackrel{\mathrm{d}}{=} T_{V 0}^{(1)}, \\
E e^{-s W_{2}}=E e^{-\eta(s) V},
\end{gathered}
$$

where $\eta(s)$ is the unique continuous solution to the equation

$$
\eta(s / c)=s+\phi(\eta(s / c))
$$


and

$$
E W_{2}=\frac{E V}{c\left(1-\rho_{1}\right)}
$$

Proof. As indicated above, $W_{2}$ is the first passage time to 0 for class 1 starting with $V$. The Laplace transform of this first passage time conditional on $V$ is given on p. 79 of Prabhu [18], while $\eta$ is characterized on p. 74. The constant $c$ in (5.4) occurs because the processing rate here is $c$ instead of 1 . By changing the measuring units, we can regard the processing rate as 1 :

$$
E e^{s A(t) / c}=e^{t \tilde{\phi}(s)}
$$

and

$$
E e^{-s W_{2} / c}=e^{-\tilde{\eta}(s) V}
$$

where

$$
\tilde{\eta}=s+\tilde{\phi}(s)
$$

Since $\tilde{\phi}(s)=\phi(s / c)$ and $\tilde{\eta}(s)=\eta(s / c)$, we obtain (5.4). Finally, (5.5) holds because $E T_{x 0}^{(1)}=$ $x / c\left(1-\rho_{1}\right)$ for each $x$; see [18].

\section{The Reduced-Service-Rate Lower Bound}

We now drop the extra assumption in Section 5 (unless specifically stated) and consider the distributions of the low-priority steady-state workload $V_{2}$ and waiting time $W_{2}$. They are hard to determine because the server-availability process $S_{2}$ in (2.4) depends on the stochastic fluctuations of the high-priority class. A convenient rough approximation is to assume that the server is continuously available to the low-priority class at a reduced rate, with the reduction accounting for the long-run average usage of the high-priority class. In particular, we call the approximation

$$
S_{2}(t) \approx S_{2}^{r}(t) \equiv\left(1-\rho_{1}\right) c t, \quad t \geq 0,
$$

the reduced-service-rate (RSR) approximation. With the RSR approximation, we can analyze the two priority classes separately, just as in a system without priorities. The RSR approximation decouples the system, making the low-priority class depend upon the high-priority class only through the offered-load parameter $\rho_{1}$.

By (2.6) and (6.1), the associated waiting-time and workload approximations are related by

$$
W_{2}^{r}(t)=\frac{V_{2}^{r}(t)}{c\left(1-\rho_{1}\right)}, \quad t \geq 0
$$


and

$$
W_{2}^{r}=\frac{V_{2}^{r}}{c\left(1-\rho_{1}\right)}
$$

with the steady-state workload being

$$
\begin{aligned}
V_{2}^{r} & =\sup _{t \geq 0}\left\{A_{2}(-t)+\left(1-\rho_{1}\right) c t\right\} \\
& =\sup _{t \geq 0}\left\{A_{2}\left(-t /\left(1-\rho_{1}\right)\right)+c t\right\},
\end{aligned}
$$

which is the formula for $V_{1}$ in $(3.2)$ with $\left\{A_{1}(t): t \geq 0\right\}$ replaced by the scaled process $\left\{A_{2}(t /(1-\right.$ $\left.\left.\left.\rho_{1}\right)\right): t \geq 0\right\}$.

It is intuitively clear that the RSR approximation is typically optimistic, i.e., that we should usually have $V_{2}^{r}$ and $W_{2}^{r}$ smaller than their counterparts $V_{2}$ and $W_{2}$. We now present some supporting evidence using stochastic comparison concepts. We say that a random variable $U_{1}$ is less than or equal to another $U_{2}$ in increasing convex order and write $U_{1} \leq_{i c x} U_{2}$ if $\operatorname{Ef}\left(U_{1}\right) \leq E f\left(U_{2}\right)$; for all nondecreasing convex real-valued functions $f$ for which the expectations are well defined; see Stoyan [22] or Chapter 4 of Baccelli and Brémaud [1]. The essential line of reasoning below goes back to Rogozin [20].

Theorem 6.1. In the general stationary model, $V_{2}^{r} \leq_{i c x} V_{2}$.

Proof. We work with the stationary versions defined in Section 2. Then

$$
E S_{2}^{*}(t)=S_{2}^{r}(t), \quad t \geq 0
$$

for all $t$, where $S_{2}^{r}(t)$ is defined in (6.1). Hence, the processes $\left\{S_{2}^{*}(t): t \geq 0\right\}$ and $\left\{S_{2}^{r}(t): t \geq 0\right\}$ are ordered by convex stochastic order; i.e., $S_{2}^{*} \geq_{c x} S_{2}^{r}$, by which we mean that

$$
E f\left(\left\{S_{i}^{*}(t): t \geq 0\right\}\right) \geq E f\left(\left\{S_{i}^{r}(t): t \geq 0\right\}\right)
$$

for all real-valued convex functions $f$ on the space of sample paths for which the expections are well define; see Remark 2 on p. 81 of Stoyan [22] and pp. 198, 220 of Baccelli and Brémaud [1] for related arguments. By (3.2), $V_{2}$ and $V_{2}^{r}$ can be written as (nonincreasing) convex real-valued functions of $\left\{S_{2}^{*}(t): t \geq 0\right\}$ and $\left\{S_{2}^{r}(t): t \geq 0\right\}$, respectively. Since nondecreasing convex real-valued functions of arbitrary convex real-valued functions are convex, we have the stated conclusion; i.e.,

$$
E g\left(V_{2}^{r}\right)=E g \circ f\left(S_{2}^{r}\right) \leq E g \circ f\left(S_{2}^{*}\right)=E g\left(V_{2}\right)
$$

for all nondecreasing convex $g$, where $f$ here denotes the convex functions taking $S_{2}^{r}$ into $V_{2}^{r}$ and $S_{2}^{*}$ into $V_{2}^{*}$. 
The $\leq_{i c x}$ ordering in Theorem 6.1 implies that $E\left(V_{2}^{r}\right)^{k} \leq E\left(V_{2}^{k}\right)$ for all $k \geq 1$. However, the $\leq_{i c x}$ ordering is weaker than ordinary stochastic order $V_{2}^{r} \leq_{s t} V_{2}$ which would hold if $\operatorname{Ef}\left(V_{2}^{r}\right) \leq E f\left(V_{2}\right)$ for all nondecreasing real-valued functions $f$. We now show that the ordering in Theorem 6.1 cannot be strengthened to stochastic order.

Example 6.1. To see that we need not have $V_{2}^{r} \leq_{s t} V_{2}$, we show that it is possible to have $P\left(V_{2}^{r}>\right.$ $0)>P\left(V_{2}>0\right)$. Our example also shows that it is possible to have $P\left(W_{2}^{r}>0\right)>P\left(W_{2}>0\right)$, so that in general we do not have $W_{2}^{r} \leq_{s t} W_{2}$ either. First, if $A_{2}(t)$ is a pure-jump process, then we always have (by Little's law applied to the server),

$$
P\left(V_{2}^{r}>0\right)=\frac{\rho_{2}}{\left(1-\rho_{1}\right) c}
$$

For our concrete example, let $c=1$ and initially let $A_{2}(t)=\rho_{2} t, t \geq 0$, corresponding to deterministic input. (We will later make $A_{2}(t)$ a pure jump process.) Let the high-priority input occur in constant lumps of size $\rho_{1}$ spaced apart according to i.i.d. random variables distributed as $\rho_{1} /\left(1+\rho_{2}\right)+X$, where $X$ is exponentially distributed with mean $1-\rho_{1} /\left(1-\rho_{2}\right)$. Thus the mean time between successive class- 1 inputs of size $\rho_{1}$ is 1 . Following each type 1 input of size $\rho_{1}$, there is a period of length $\rho_{1}$ during which the server works on this input. At the end of this period there is $\rho_{1} \rho_{2}$ class- 2 work. The server then takes $\rho_{1} \rho_{2} /\left(1-\rho_{2}\right)$ time to clear this class- 2 work. The remainder of the interval before the next class-1 input, of length $X$, the server is processing only the class-2 input. Hence, for this model (using regenerative analysis),

$$
P\left(V_{2}>0\right)=\frac{\rho_{1}}{1-\rho_{2}}
$$

so $P\left(V_{2}^{r}>0\right)>P\left(V_{2}>0\right)$ if and only if $\rho_{2}\left(1-\rho_{2}\right)>\rho_{1}\left(1-\rho_{1}\right)$. Since we must have $\rho_{1}+\rho_{2}<1$ for stability, this inequality holds whenever $\rho_{1}<\rho_{2}$. For a somewhat extreme case, let $\rho_{1}=0.1$ and $\rho_{2}=0.5$. Then

$$
P\left(V_{2}^{r}>0\right)=\frac{5}{9}>\frac{1}{5}=P\left(V_{2}>0\right)
$$

Now we have to make $A_{2}(t)$ a pure jump process behaving approximately like deterministic input. For this purpose, let $A_{2}^{(\epsilon)}$ denote the input process having jumps of size $\epsilon \rho_{2}$ spaced apart by i.i.d. random variables distributed as $\epsilon \rho_{2}+\epsilon Y$, where $Y$ is an exponential random variable with mean $1-\rho_{2}$. Let $V^{(\epsilon) r}$ denote the RSR approximation associated with $A_{2}^{(\epsilon)}$. As $\epsilon \rightarrow 0, A_{2}^{(\epsilon)}(t)$ approaches deterministic input, so that $P\left(V_{2}^{(\epsilon) r}>0\right) \rightarrow P\left(V_{2}>0\right)$ in (6.7), but (6.6) holds for all $\epsilon$. Hence, the counterexample in (6.8) holds for all sufficiently small $\epsilon$. Finally, this example also serves for the 
steady-state (virtual) waiting times, because $P\left(W_{2}^{r}>0\right)=P\left(V_{2}^{r}>0\right)$ and $P\left(W_{2}>0\right)=P\left(V_{2}>0\right)$ here.

We have yet to establish a result corresponding to Theorem 6.1 for the waiting times. However, we can establish an exact representation for $W_{2}$ in terms of $V$ when the high-priority class input has independent increments, as assumed in Section 5. We now show that $W_{2}^{r}$ is a lower bound for $W_{2}$ under this extra condition

Theorem 6.2. Under the conditions of Theorem 5.1,

$$
W_{2} \geq_{c x} \frac{V}{c\left(1-\rho_{1}\right)} \geq_{i c x} \frac{V_{2}^{a}}{c\left(1-\rho_{1}\right)}=W_{2}^{r}
$$

so that $W_{2} \geq_{i c x} W_{2}^{a}$.

Proof. Since $E T_{x 0}^{(1)}=x / c\left(1-\rho_{1}\right)$ for each $x$,

$$
E\left(T_{V 0}^{(1)} \mid V\right)=\frac{V}{c\left(1-\rho_{1}\right)} .
$$

Thus, for any convex $g$,

$$
E\left[g\left(T_{V 0}^{(1)}\right) \mid V\right] \geq g\left(V / c\left(1-\rho_{1}\right)\right) \quad w . p .1
$$

and

$$
E g\left(T_{V 0}^{(1)}\right) \geq E g\left(V / c\left(1-\rho_{1}\right)\right)
$$

i.e., $T_{V 0}^{(1)} \geq_{c x} V / c\left(1-\rho_{1}\right)$. Hence,

$$
W_{2} \stackrel{d}{=} T_{V 0}^{(1)} \geq_{c x} \frac{V}{c\left(1-\rho_{1}\right)}>\frac{V_{2}}{c\left(1-\rho_{1}\right)} \geq_{i c x} \frac{V_{2}^{r}}{c\left(1-\rho_{1}\right)}=W_{2}^{r},
$$

where we have used Theorem 6.1 in the penultimate step.

The RSR approximation is not only a bound. It also arises as a special case in which class-1 input is a fluid or as a limit in which the class-1 input approaches a fluid input. This implies that the bound is sharp, i.e., is attained in some cases.

We now show that the resulting effective bandwidth approximation is optimistic.

Theorem 6.3. In the general stationary model,

$$
\left(1-\rho_{1}\right) c \theta=\psi_{S_{2}^{r}}(\theta) \leq \psi_{S_{2}}(\theta) \text { for all } \theta
$$

so that for the workload asymptotic decay rates in Theorem 3.1 are ordered by

$$
\theta_{2}^{* r} \geq \theta_{2}^{*}
$$


and, for any $\eta_{2}^{*}>0$, the effective capacities are ordered by

$$
C_{2}^{r} \equiv \frac{-\psi_{S_{2}^{r}}\left(-\eta_{2}^{*}\right)}{\eta_{2}^{*}} \geq \frac{-\psi_{S_{2}}\left(-\eta_{2}^{*}\right)}{\eta_{2}^{*}} \equiv C_{2}
$$

Proof. The convex order $S_{2}^{r} \leq_{c x} S_{2}$ used in the proof of Theorem 6.1 implies that $E e^{\theta S_{2}^{r}(t)} \leq$ $E e^{\theta S_{2}^{*}(t)}$ for all $\theta$ and $t$ from which (6.11) follows immediately. In turn (6.12) and 6.16) follow easily from (6.11) and (4.13).

If we use the RSR approximation, then the admission criteria in (4.14) become

$$
\begin{gathered}
\sum_{j=1}^{J_{1}} e_{1 j} n_{i j}=\sum_{j=1}^{J_{1}} \frac{\psi_{A_{1 j}}\left(\eta_{1}^{*}\right)}{\eta_{1}^{*}} n_{1 j} \leq c \\
\sum_{j=1}^{J_{2}} e_{2 j} n_{2 j}=\sum_{j=1}^{J_{2}} \frac{\psi_{A_{2 j}}\left(\eta_{2}^{*}\right)}{\eta_{2}^{*}} n_{2 j} \leq c\left(1-\rho_{1}\right),
\end{gathered}
$$

where $\rho_{1}$ in (6.15) is the utilization of the $J_{1}$ class- 1 sources, and the target parameters $\eta_{i}^{*}$ are as in (4.7) with the constraints in (4.1) to be met for large $b_{i}, i=1,2$. Since $\rho_{1}=\sum_{j=1}^{J_{1}} \rho_{1 j} n_{1 j},(6.15)$ can be written as

$$
\sum_{j=1}^{J_{1}} c \rho_{1 j} n_{1 j}+\sum_{j=1}^{J_{2}} e_{2 j} n_{2 j} \leq c .
$$

The pair of constraints (6.14) and (6.16) form a linear feasible set.

\section{A Further Lower Bound and Heavy Tails}

In this section we apply [8] to obtain a stochastic lower bound for $V_{2}^{r}$ that enables us to obtain a sufficient condition for $V_{2}$ to have a heavy-tailed distribution. Following [8], let the low-priority input process be a general stochastic fluid input process determined by a stationary environment process $\left\{Z_{2}(t): t \geq 0\right\}$. We assume that the environment process spends alternating positive times $X_{1}, Y_{1}, X_{2}, Y_{2}, \ldots$ in states such that the input is above and below a high rate $r_{2}$. We assume that $\left\{\left(X_{n}, Y_{n}\right)\right\}$ is a stationary sequence with $E X_{n}<\infty$ and $E Y_{n}<\infty$.

Let $G$ be the cumulative distribution function (cdf) of a high-activity period $X_{n}$ and let $G^{c}(t) \equiv$ $1-G(t)$ be the associated complementary cdf (ccdf). Let $G_{e}$ be the associated stationary-excess cdf, defined by

$$
G_{e}(t)=\frac{1}{E X_{1}} \int_{0}^{t} G^{c}(u) d u, \quad t \geq 0
$$

Theorem 7.1. (from [8]) Under the assumptions above, if $r_{2}>c\left(1-\rho_{1}\right)$, then

$$
P\left(V_{2}^{r}>t\right) \geq F^{c}(t) \equiv\left(\frac{E X_{1}}{E X_{1}+E Y_{1}}\right) G_{e}^{c}\left(t /\left(r_{2}-c\left(1-\rho_{1}\right)\right)\right)
$$


so that

$$
\limsup _{t \rightarrow \infty} \frac{P\left(V_{2}>t\right)}{G_{e}^{c}\left(t /\left(r_{2}-c\left(1-\rho_{i}\right)\right)\right)} \geq \frac{E X_{1}}{E X_{1}+E Y_{1}}>0
$$

Proof. Inequality (7.2) is Theorem 1 of [8]. Since $V_{2} \geq_{i c x} V_{2}^{r}$, we have

$$
\int_{t}^{\infty} P\left(V_{2}>u\right) d u \geq \int_{0}^{\infty} P\left(V_{2}^{r}>u\right) d u \text { for all } t
$$

see p. 8 of [22], which implies (7.3).

Property (7.3) can be interpreted as saying that the ccdf of $V_{2}$ has a heavier tail than the ccdf $G_{e}^{c}$. For example, if

$$
\lim _{t \rightarrow \infty} t^{\eta} G_{e}^{c}(t)=\alpha
$$

where $\eta$ and $\alpha$ are positive constants, then Theorem 7.1 implies that

$$
\limsup _{t \rightarrow \infty} t^{\eta} P\left(V_{2}>t\right)>0
$$

\section{The Empty-Buffer Upper Bound}

The empty-buffer bound is based on considering what the class- 2 departure process would be if there were never any accumulation of class-1 workload, as would occur with continuous deterministic input with $\rho_{1}<1$. If class 1 never had workload, i.e., if $V_{1}(t)=0$ for all $t$, then we would have $D_{1}(t)=A_{1}(t)$ and $S_{2}(t)=c t-A_{1}(t)$. Thus, we define the empty-buffer bound to be

$$
S_{2}(t) \approx S_{2}^{e}(t) \equiv c t-A_{1}(t)
$$

Suppose that we now consider the departure process starting out empty. In that case $D_{1}(t) \leq$ $A_{1}(t), t \geq 0$, so that

$$
S_{2}(t) \geq S_{2}^{e}(t), t \geq 0
$$

Indeed, by (2.2)-(2.4),

$$
X_{2}^{e}(t)=A_{1}(t)+A_{2}(t)-c t, \quad t \geq 0,
$$

so that the empty-buffer bound is equivalent to approximating the class- 2 workload process by the aggregate workload, i.e.,

$$
V_{2}^{e}(t)=V(t) \equiv V_{1}(t)+V_{2}(t), \quad t \geq 0 .
$$

Hence, we have the following elementary comparison result.

Theorem 8.1. In the general stationary model, $V_{2} \leq_{s t} V_{2}^{e}=V$. 
Proof. Consider the system starting out empty. Clearly the sample paths are ordered: $V_{2}(t) \leq$ $V(t)=V_{2}^{e}(t)$ for all $t \geq 0$. Since stochastic order is preserved under convergence in distribution, the conclusion follows.

The associated empty-buffer effective-bandwidth (EBEB) approximation is also conservative. Paralleling Theorem 6.3, we have the following elementary result.

Theorem 8.2. In the general stationary model,

$$
\psi_{S_{2}^{e}}(\theta) \geq \psi_{S_{2}}(\theta) \text { for all } \theta<0
$$

so that the workload asymptotic decay rates in Theorem 3.1 are ordered by

$$
\theta_{2}^{* e} \leq \theta_{2}^{*}
$$

and, for all $\eta_{2}^{*}>0$, the effective capacities are ordered by

$$
C_{2}^{e} \equiv \frac{-\psi_{S_{2}^{e}}\left(-\eta_{2}^{*}\right)}{\eta_{2}^{*}}=c-\frac{\psi_{A_{1}}\left(\eta_{2}^{*}\right)}{\eta_{2}^{*}} \leq c-\frac{\psi_{D_{1}}\left(\eta_{2}^{*}\right)}{\eta_{2}^{*}}=-\frac{\psi_{S_{2}}\left(-\eta_{2}^{*}\right)}{\eta_{2}^{*}} \equiv C_{2} .
$$

At first glance, the empty-buffer bound may seem very crude, but it can be surprisingly accurate. It often happens that the bulk of the workload is low-priority work. Indeed, in support of the emptybuffer approximation, we point out that it is asymptotically exact as $\rho_{2} \rightarrow 1-\rho_{1}$ for any $\rho_{1}$ (in heavy traffic), see [25]. In that limit, the total workload is growing, being of order $O(1 /(1-\rho))$, where $\rho=\rho_{1}+\rho_{2} \rightarrow 1$, while the class-1 workload remains unchanged. Hence there definitely are scenarios where the empty-buffer bound provides an excellent approximation.

Paralleling (6.14) and (6.15), the admission criteria with the empty buffer approximation are (6.14) and

$$
\sum_{j=1}^{J_{2}} e_{2 j} n_{2 j}=\sum_{j=1}^{J_{2}} \frac{\psi_{A_{2 j}}\left(\eta_{2}^{*}\right)}{\eta_{2}^{*}} n_{2 j} \leq c-\frac{\psi_{A_{1}}\left(\eta_{2}^{*}\right)}{\eta_{2}^{*}}=C_{2}^{e}
$$

Since

$$
\frac{\psi_{A_{1}}\left(\eta_{2}^{*}\right)}{\eta_{2}^{*}}=\sum_{j=1}^{k_{1}} \frac{\psi_{A_{1 j}}\left(\eta_{2}^{*}\right) n_{1 j}}{\eta_{2}^{*}}
$$

the two constraints (6.14) and (8.6) are fully linear. Note that $\psi_{A_{1 j}}\left(\eta_{2}^{*}\right) / \eta_{2}^{*}$ in (8.7) is similar to the effective bandwidth of a class- 1 source of type $j$, except $\eta_{2}^{*}$ is present as opposed to $\eta_{1}^{*}$. We call $\psi_{A_{1 j}}\left(\eta_{2}^{*}\right) / \eta_{2}^{*}$ the effective bandwidth of a $(1, j)$ source as seen by class 2 , and denote it $e_{1 j}^{2}$. Thus the admission criteria for the effective-bandwidth empty buffer approximation can be written as:

$$
\sum_{j=1}^{J_{1}} e_{1 j} n_{1 j} \leq c
$$




$$
\sum_{j=1}^{J_{1}} e_{1 j}^{2} n_{1 j}+\sum_{j=1}^{J_{2}} e_{2 j} n_{2 j} \leq c .
$$

\section{An Illustrative Example}

The reduced service rate (RSR) and empty buffer (EB) approximations provide upper and lower bounds respectively for the priority-2 effective capacity, $C_{2}$, (4.13). In particular, from (6.11), (6.13), and (8.5),

$$
C_{2}^{r}=\left(1-\rho_{1}\right) c \geq C_{2} \geq c-\frac{\psi_{A_{1}}\left(\eta_{2}^{*}\right)}{\eta_{2}^{*}}=C_{2}^{e} .
$$

Thus, the difference $C_{2}^{r}-C_{2}^{e}$ is an upper bound on the error if either $C_{2}^{r}$ or $C_{2}^{e}$ is used as an approximation for $C_{2}$. For a perspective on the size of this error, it is natural to normalize by the aggregate capacity $c$, obtaining the normalized error bound, denoted

$$
E \equiv\left(C_{2}^{r}-C_{2}^{e}\right) / c
$$

From (9.1), $E$ can be expressed as

$$
E=\frac{\psi_{A_{1}}\left(\eta_{2}^{*}\right)}{c \eta_{2}^{*}}-\rho_{1}
$$

or equivalently from $(3.1)$

$$
E=\frac{1}{c}\left[\frac{\psi_{A_{1}}\left(\eta_{2}^{*}\right)}{\eta_{2}^{*}}-\lim _{t \rightarrow \infty} \frac{A_{1}(t)}{t}\right]
$$

Note that the normalized error bound depends on the aggregate high-priority arrival process, $A_{1}(t)$, and the low-prioirty performance parameters represented by $\eta_{2}^{*}=-\log \left(p_{2}\right) / b_{2},(4.7)$. Also note that in the boundary case where the priority- 1 arrival process is a constant rate fluid, $E$ equals zero.

For the application to packet communication networks, one would like the normalized error bound to be less than the noise in the traffic model, $A_{i}(t)$. Often the traffic models deviate from reality by more than $10 \%$, particularly if a forecast is involved. Thus, if $E$ is less than $10 \%$ then the error from the RSR or EB approximations is within the noise of the model.

As a first example, consider the case of an ATM network where the high-priority class supports constant-bit-rate (CBR) connections. As mentioned in Section 5, due to network jitter and the lack of synchronization across the connections, the superposition of the jittered CBR streams can be modelled, often conservatively, as a Poisson process. If $A_{1}(t)$ is a compound Poisson process with 
Poisson rate $c \rho_{1}$ and component unit-size jumps (where a jump represents the arrival of an ATM cell, which has a constant size), then $\psi_{A_{1}}(\theta)=c \rho_{1}\left(e^{\theta}-1\right)$ and

$$
E=\rho_{1}\left[\frac{e^{\eta_{2}^{*}}-1}{\eta_{2}^{*}}-1\right]=\frac{\rho_{1} \eta_{2}^{*}}{2}+O\left(\eta_{2}^{* 2}\right) .
$$

For a particular example, if the priority-2 buffer threshold, $b_{2}$, is 500 and the probability that the work in system exceeds $b_{2}$ should be no more than $p_{2}=10^{-3}$, then $\eta_{2}^{*}$ is 0.0138 . If $\rho_{1}$ is 0.50 , then $E$ is 0.003 , which is well within the noise of the traffic models.

As a second example, suppose the aggregate prioirty-1 arrival process is a two-state Markov modulated Poisson process (MMPP) where one state is on while the other is off, and hence the process is also equivalent to an interrupted Poisson process. The MMPP has rate matrix

$$
\Lambda=\left(\begin{array}{cc}
\lambda_{1} & 0 \\
0 & 0
\end{array}\right)
$$

and infinitesimal generator

$$
M=\left(\begin{array}{cc}
-r_{1} & r_{1} \\
r_{2} & -r_{2}
\end{array}\right)
$$

and where each arrival adds one unit of work. The asymptotic-decay-rate function can be expressed in closed form:

$$
\psi_{A_{1}}(\theta)=\left(-\alpha+\sqrt{\alpha^{2}+4 \lambda_{1} r_{2}\left(e^{\theta}-1\right)}\right) / 2
$$

where $\alpha=r_{1}+r_{2}-\lambda_{1}\left(e^{\theta}-1\right)$.

For a particular example, suppose that $\lambda_{1}, r_{1}$, and $r_{2}$ are specified by the mean rate of $A_{1}(t)$, $\lambda_{1} r_{2} /\left(r_{1}+r_{2}\right)$, equaling 0.01 , and the fraction of time on, $r_{2} /\left(r_{1}+r_{2}\right)$, equaling $0.1,0.05$, or 0.01 , and the mean number of arrivals during an on period (mean burst size), $\lambda_{1} / r_{1}$ equaling 10 or 100 , and the capacity $c=1$. For this arrival process and for priority-2 performance parameters of $p_{2}$ $=10^{-3}$ and $b_{2} \in\{100,500,1000\}$, Table 1 reports the resulting normalized error bound, $E$. The parameter values were chosen to show where the RSR and EB approximations begin to perform poortly. When the mean burst size is as big as the buffer threshold, as when they both are 100, $E$ is relatively large, particularly for the bursty case where the fraction of time on is only 1 percent. However, for low priority traffic in packet networks, where significant queueing can be expected, the buffer should be an order of magnitude bigger than the mean burst size. For these cases, the normalized error bound is less than $10 \%$, which is within the noise of typical traffic models.

The RSR and EB approximations for the effective capacity $C_{2}$ can be used to approximate the admissible sets for the number of priority- 1 and priority- 2 connections that can be admitted while satisfying the performance parameters. We use the RSR and EB approximations derived 


\begin{tabular}{|c|c|c|c|}
\hline $\begin{array}{c}\text { Fraction } \\
\text { of time } \\
\text { ON }\end{array}$ & $\begin{array}{c}\text { Mean } \\
\text { burst } \\
\text { size }\end{array}$ & $\begin{array}{c}\text { Buffer } \\
\text { threshold } \\
b_{2}\end{array}$ & $\begin{array}{c}\text { Normalized } \\
\text { error } \\
\text { bound, } E\end{array}$ \\
\hline .1 & 10 & 100 & .011 \\
.1 & 10 & 500 & .0013 \\
.1 & 10 & 1000 & .00062 \\
.1 & 100 & 100 & .079 \\
.1 & 100 & 500 & .030 \\
.1 & 100 & 1000 & .010 \\
& & & \\
.05 & 10 & 100 & .016 \\
.05 & 10 & 500 & .0015 \\
.05 & 10 & 1000 & .00070 \\
.05 & 100 & 100 & .17 \\
.05 & 100 & 1000 & .014 \\
.05 & 100 & 500 & .061 \\
& & & \\
.01 & 10 & 100 & .023 \\
.01 & 10 & 500 & .0017 \\
.01 & 10 & 1000 & .00076 \\
.01 & 100 & 100 & .88 \\
.01 & 100 & 500 & .29 \\
.01 & 100 & 1000 & .020 \\
\hline
\end{tabular}

Table 1: Normalized error bound $E$, given priority-1 arrival process is an on/off MMPP with mean rate 0.01 , and various fraction of $\mathrm{ON}$ times and mean burst sizes, and given priority-2 performance parameters $p_{2}=10^{-3}$ and various buffer thresholds $b_{2}$. 
herein to examine these admissible sets in detail in [2]. As the RSR approximation gives an upper bound on $C_{2}$, it yields an optimistic approximation for the admissible set, and likewise since the EB approximation gives a lower bound on $C_{2}$, it yields a conservative approximation. When the priority-2 performance parameters are significantly looser than those for priority $1\left(\eta_{2}^{*}\right.$ an order of magnitude smaller than $\eta_{1}^{*}$ ), then for a given number of priority-1 connections, the RSR and EB estimates for the number of admissible priority-2 connections are often close - equaling the same integer value, or differing by 1 or 2 . 


\section{References}

[1] F. Baccelli and P. Brémaud, Elements of Queueing Theory, Springer-Verlag, New York, 1994.

[2] A. W. Berger and W. Whitt, Effective bandwidths with priorities, IEEE/ACM Trans. Networking 6 (1998) 447-460.

[3] A. W. Berger and W. Whitt, Extending the effective bandwidth concept to networks with priority classes, IEEE Communications Magazine, August (1998), 78-83.

[4] A. A. Borovkov, Stochastic Processes in Queueing Theory, Springer-Verlag, New York, 1976.

[5] L. Breiman, Probability, Addison-Wesley, Reading, MA, 1968.

[6] R. Cáceres, P. G. Danzig, S. Jamin and D. J. Mitzel, Characteristics of wide-area TCP/IP conversations, Computer Communications Review 21 (1991) 101-112.

[7] C. S. Chang and J. A. Thomas, Effective bandwidths in high-speed digital networks, IEEE J. Sel. Areas Commun. 13 (1995) 1091-1100.

[8] G. L. Choudhury and W. Whitt, Long-tail buffer-content distributions in broadband networks, Performance Evaluation 30 (1997) 177-190.

[9] G. de Veciana, G. Kesidis and J. Walrand, Resource management in wide-area ATM networks using effective bandwidths, IEEE J. Sel. Areas Commun. 13 (1995) 1081-1090.

[10] N. G. Duffield and N. O'Connell, Large deviations and overflow probabilities for the general single-server queue, with applications, Math. Proc. Camb. Phil. Soc. 118 (1995) 363-374.

[11] A. I. Elwalid and D. Mitra, Analysis, approximations and admission control of a multi-service multiplexing system with priorities, Proc. IEEE Infocom '95, pp. 463-472, 1995.

[12] A. Feldmann, A. C. Gilbert, W. Willinger and T. G. Kurtz, The changing nature of network traffic: scaling phenomena, Computer Communcations Review, 1998, to appear.

[13] P. W. Glynn and W. Whitt, Ordinary CLT and WLLN versions of $L=\lambda W$, Math. Oper. Res. 13 (1988) 674-692.

[14] P. W. Glynn and W. Whitt, Logarithmic asymptotics for steady-state tail probabilities in a single-server queue, Studies in Applied Probability, Essays in Honour of Lajos Takács, eds. J. Gani and J. Galambos, Applied Probability Trust, Sheffield, 1994, 131-156. 
[15] F. Kelly, Notes on effective bandwidths, in Stochastic Networks, Clarendon Press, Oxford, 1996, 141-168.

[16] V. G. Kulkarni and N. Gautam, Admission control of multi-class traffic with service priorities in high-speed networks, Queueing Systems 27 (1997) 79-97.

[17] W. E. Leland, M. S. Taqqu, W. Willinger and D. V. Wilson, On the self-similar nature of Ethernet traffic, IEEE/ACM Trans. Networking 2 (1994) 1-15.

[18] N. U. Prabhu, Stochastic Storage Processes, Springer-Verlag, New York, 1980.

[19] A. A. Puhalskii and W. Whitt, Functional large deviation principles for waiting and departure processes, Prob. Eng. Inf. Sci. 12 (1998) 479-507.

[20] B. A. Rogozin, Some extremal properties in the theory of mass service, Theor. Prob. Appl. 11 (1966) 44-151.

[21] K. W. Ross, Multiservice Loss Models for Broadband Telecommunication Networks, PrenticeHall, Englewood Cliffs, NJ, 1995.

[22] D. Stoyan, Comparison Methods for Queues and Other Stochastic Models, Wiley, New York, 1983.

[23] A. Sugahara, T. Takine, Y. Takahashi and T. Hasegawa, Analysis of a nonpreemptive priority queue with SPP arrivals of high class, Perf. Eval. 21 (1995) 215-238.

[24] T. Takine and T. Hasegawa, The workload in the MAP/G/1 queue with state-dependent services: its application to a queue with preemptive resume priority, Stochastic Models 10 (1994) 183-204.

[25] W. Whitt, Weak convergence theorems for priority queues: preemptive-resume discipline, $J$. Appl. Prob. 8 (1971) 74-94.

[26] W. Whitt, Some useful functions for functional limit theorems, Math. Oper. Res. 5 (1980) $67-85$.

[27] W. Whitt, Tail probabilities with statistical multiplexing and effective bandwidths in multiclass queues, Telecommunication Systems 2 (1993) 71-107.

[28] R. W. Wolff, Poisson arrivals see time averages, Operations Res. 30 (1982) 223-231. 
[29] J. Zhang, Performance study of Markov modulated fluid flow models with priority traffic, Proc. IEEE Infocom '93 1993, 10-17. 